\title{
Gingival Hemangiosarcoma of the Left Mandible in a Dog
}

\begin{abstract}
Keywords: Dog; Mandible; Gingiva; Hemangiosarcoma; Hemimandibulectomy

Abstract

A ten yearold Dogo Argentino cross, waspresented with a fast growing mass in the left mandible. The dog underwent hemimandibulectomy and cheiloplasty. No chemotherapeutic regimes were administered, post-operatively. The histopathological findings were suggestive of hemangiosarcoma. The a nimal survived with no signs of metastasis or recurrence for 5 months, when local recurrence was identified and radiographic examination revealed lung metastasis. Consequently, the owner decided to euthanize the dog. Mandibular hemangiosarcoma is aggressive both locally and systemically as it recurred and metastasized, even after extensive exc ision. To the a uthors' knowledge this is a rare case of mandibular hemangiosarcoma reported in the dog.
\end{abstract}

\section{Introduction}

Hemangiosarcoma is a highly malignant neoplasm of vascular endothelial origin which is reported to be locally invasive and rapidly metastasizing, with the exception of primary canine subcutaneous hemangiosarcoma [1].

Hemangiosarcoma occurs more frequently in dogs than in other domestic species with an overall prevalence of $2 \%$ of all tumors accounted in dogs [2-4]. Most studies indicate that the spleen is the most frequently affected organ [2,5]. Other primary sites of involvement in the dog include the right atrium, liver, skin and subcutis [2,4-7]. Bones such as the proximal humerus, femur, ribs and vertebrae can also be affected [8].

No breed or sex predilection has been proven [2], although many reports have shown an increased prevalence in German Shepherds and males $[3,5,9,10]$. The mean age of affected dogs at the time of diagnosis is estimated to be 9 to 11 years $[3,5]$, even though one year old or younger animals have been presented $[10,11]$.

In this case report, we present a case of mandibular hemangiosarcoma in a dog.

\section{Case Report}

A 10 year old, $39 \mathrm{~kg}$, intact male Dogo Argentino cross, was referred for a fast growing oral mass. The owner had noticed the mass a month prior to presentation, due to continuous salivation, bleeding and halitosis. Since then, the tumor manifested surprisingly rapid growth and the animal developed anorexia and significant weight loss. On presentation, the clinical examination revealed tachypnea, normal heart rate and body temperature, normal thorax and heart auscultation, depression and emaciation. A facial swelling was obvious on the left side of the mandible. The overlying skin appeared normal. Because of the depression and the swelling, a cranial nerve examination was performed, which was normal. The oral examination was performed under deep sedation and manifested a growth of $10 \times 10 \mathrm{~cm}$ diameter. The tumor covered both the buccal

\section{Journal of}

Veterinary Science \& Medicine

\section{Serafim A Papadimitriou ${ }^{1 *}$, Anthi Anatolitou ${ }^{1}$ Georgia Brellou ${ }^{2}$, Maria I Kouki ${ }^{1}$ and loannis} Vlemmas $^{2}$

${ }^{1}$ Companion Animal Clinic, Faculty of Veterinary Medicine, Aristotle University of Thessaloniki, Greece

${ }^{2}$ Laboratory of Pathology, Faculty of Veterinary Medicine, Aristotle University of Thessaloniki, Greece

\section{Address for Correspondence}

Serafim A Papadimitriou, 11 St Voutyra str, Thessaloniki 54627 Greece, Tel: (0030) 2310994575; Fax: (0030) 2310994449; E mail: serpap@vet.auth.gr Copyright: @ 2014 Papadimitriou SA, et al. This is an open access article distributed under the Creative Commons Attribution License, which permits unrestricted use, distribution, and reproduction in any medium, provided the original work is properly cited.

Submission: 20 January 2014

Accepted: 24 January 2014

Published: 27 January 2014

Reviewed \& Approved by: Dr. Mark Brown

Professor and faculty in the Department of Clinical Sciences at

Colorado State University, USA

and the lingual side of the mandible, extending between the second premolar (306) and second molar (311) teeth (Figure 1). Even though the tumor's mediolateral dimension was very extensive, it seemed to arise solely from the gingival tissue without involving the sublingual and buccal mucosa. Macroscopically, the mass did not appear to cross the midline. On gross examination, it was friable, ulcerated and bled instantly. The oral examination revealed an absent left fourth mandibular premolar (308) and severe gingival recession and mobility of the adjacent first molar tooth (309). The rest of the teeth seemed normal with strong gingival attachments, suggesting damage to the underlying alveolar bone of 308 and 309. Tonsils were slightly swollen and the lymph nodes of the area (parotid, mandibular) were of normal size and consistency. No other pathology was noticed in the oral cavity.

Hematologic and biochemical analysis revealed no abnormalities, except for a hematocrit $35.9 \%$ (normal: $37.1 \%-55 \%$ ) probably due to continuous hemorrhage of the mass. Thoracic radiography and abdominal ultrasonography showed no other sites of tumor growths. Mandible radiography revealed marked osteolysis of the mandible between 308 and 309. A fine needle aspiration of the left mandibular lymph node revealed only a mild inflammatory reaction. Incisional biopsy and computed tomography scan was proposed prior to surgery in order to obtain a definitive diagnosis and evaluate the course of therapy but the owner did not consent due to financial restraints. The surgery was planned to take place the following day.

The animal was premedicated with dexmedetomidine $175 \mu \mathrm{g} / \mathrm{cm}^{2}$ (Dexdomitor, Pfizer) and morphine $0.3 \mathrm{mg} / \mathrm{kg}$ im (Morphine $\mathrm{HCl}$ ) and Clindamycin $11 \mathrm{mg} / \mathrm{kg}$ iv (Dalacin C, Pfizer). General anesthesia was induced using propofol $2 \mathrm{mg} / \mathrm{kg}$ iv (Propofol, Fresenius Kabi) and after endotracheal intubation, was maintained with isoflurane (Isofluran, Abbott) delivered in oxygen.

A left commissurotomy was performed in order to gain better access in the oral cavity using a scalpel blade no 10. Hemorrhage was controlled with electrocautery and finger pressure. Due to lack 
Citation: Papadimitriou SA, Anatolitou A, Brellou G, Kouki MI, Vlemmas I. Gingival Hemangiosarcoma of the Left Mandible in a Dog. J Veter Sci Med. 2014;2(1): 4.

of preoperative diagnosis concerning the type of the oral tumor, wide excision was decided by the surgeons (SP. AA, MK). Based on the extension of the mass (306-311), left hemimandibulectomy by disarticulation of the temporomandibular joint was performed in order to gain clear surgical margins avoiding the tongue frenulum, salivary papillae and sublingual vessels (Figure 2). The wound was irrigated with isotonic saline and the remaining sublingual mucosa was apposed with the buccal mucosa in a simple interrupted pattern (Monocryl 4/0, Ethicon). The commissure was sutured in three layers (oral mucosa, muscle and connective tissue, and skin) (Vicryl 3/0, Ethicon) (Figure 3).

Post-operatively, metronidazole and spiramycine 4 tablets SID (Stomorgyl, Merial), amoxycillin with clavulanic acid $22 \mathrm{mg} / \mathrm{kg}$ BID (Synulox, Pfizer) were administered for ten days and carprofen $2 \mathrm{mg} / \mathrm{kg}$ BID (Rimadyl, Pfizer) for four days. Due to the animal's aggressiveness and his ability to consume soft food immediately after surgery, he was dismissed the first postoperative day.

Histopathology revealed the presence of ulcerated mandible and oral mucosa accompanied by inflammatory cell infiltration consisting mostly of neutrophilic polymorphonuclear cells. Randomly distributed necrotic foci and extensive hemorrhages, were observed in all specimens (Figure 4a). In the lamina propria were detected multiple, usually red blood filled, channels that resembled blood vessels of several diameter (Figure 4a, Figure $4 \mathrm{~b}$ and Figure 4c). One and less commonly two layers of rather atypical and pleomorphic endothelial cells with numerous atypical mitotic figures, lined those vascular channels. The neoplastic cells were spindle or ovoid in shape and contained hyperchromatic nuclei (Figure 4b, Figure 4c). Similar cells were seen in a fibrous stroma that separated the above channels. The stroma varied from a delicate fibrous network to areas with fibroma-like features (Figure 4c).

At 2 weeks after discharge, there was partial wound dehiscence and mild lateral protrusion of the tongue. No mandibular drifting or interference of the arcades was observed. The animal could easily consume soft food and had gained weight. The owner declined the proposed chemotherapy due to financial restraints.

Next examination, 2 months post operatively, revealed excellent

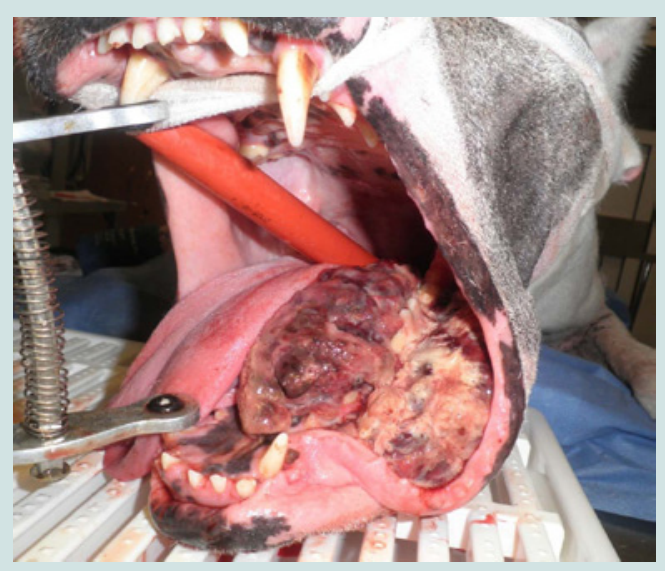

Figure 1: Oral examination revealed a mass $10 \times 10 \mathrm{~cm}$ covering the bucca and the lingual side of the mandible, extending between 306 and 311 .
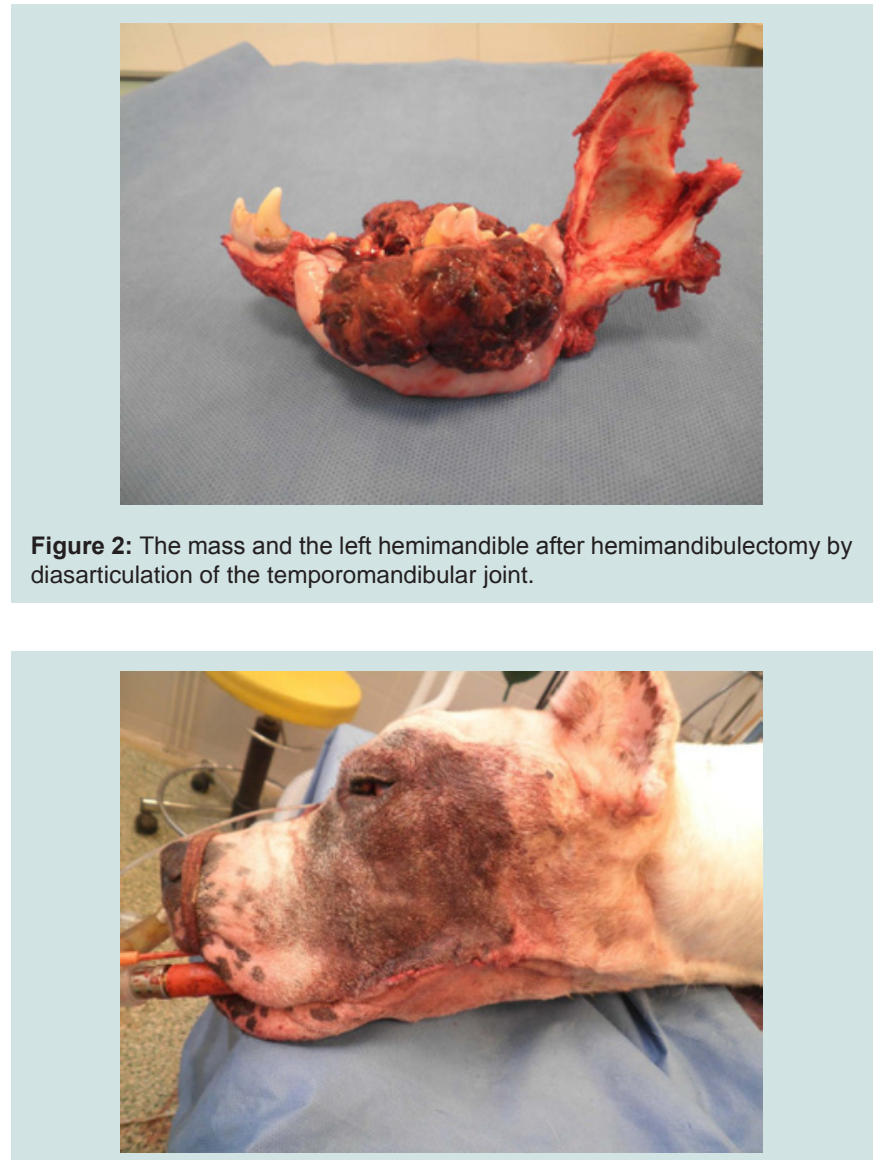

Figure 3: The dog after left hemimandibulectomy and cheiloplasty.

wound healing with no visual evidence of tumor recurrence and an accepted cosmetic appearance. The dog's physical condition was very good and he was able to consume dry food.

At 5 months, the dog was admitted again for salivation, oral bleeding and anorexia. Oral examination revealed a gross recurrence of the neoplasm on the palatopharyngeal arch (Figure 5). Moreover, lung metastasis was evident on thoracic radiographs. Due to poor prognosis the owner decided euthanasia.

During necropsy, recurrence of the primary mandibular tumor and grossly visible metastases in multiple organs, were detected. Randomly distributed and varying in size, dark red, round sometimes multilobular, blood filled formations involving the lungs, omentum, perirenal adipose tissue, kidneys, liver and spleen were observed in deep and superficial locations (Figure 6). Histopathology confirmed the angiosarcomatous nature of the metastatic masses. The findings resembled those detected in the mandible's sections (Figure $4 \mathrm{~d}$ ).

\section{Discussion}

Neoplasia of the oral and pharyngeal cavities is the fifth most common canine malignancy, with squamous cell carcinoma and malignant melanoma being the most frequent [12]. Most oral tumors arise from the gingiva in dogs. Hemangiosarcoma is a malignant neoplasm, the occurrence of which is extremely rare in the oral cavity [13]. In the veterinary literature, there are 6 reported cases of 
Citation: Papadimitriou SA, Anatolitou A, Brellou G, Kouki MI, Vlemmas I. Gingival Hemangiosarcoma of the Left Mandible in a Dog. J Veter Sci Med. 2014;2(1): 4.

glossal hemangiosarcoma concerning dogs [14,15] and one case of gingival maxillary hemangiosarcoma [16] Other types of malignant oropharyngeal tumors encountered in dogs are: fibrosarcoma, acanthomatous ameloblastoma, adenocarcinoma, undifferentiated carcinoma, lymphosarcoma, osteosarcoma, transmissible venereal tumor, mast cell tumor, neuroendocrine cell tumor and granular cell myoblastoma [12].

Visceral hemangiosarcoma is rarely curable and long-term prognosis is poor. Cutaneous hemangiosarcomas seems to have slightly better prognosis and time of survival $[6,14]$.

Surgical excision is the most effective means of treatment for oral tumors. A $2 \mathrm{~cm}$ margin of normal tissue should be removed with the tumor [12]. Radiotherapy offers the advantage of treating larger areas of tissue surrounding the tumor than may be possible by surgery. Local lymph nodes can also be included in the treatment fields where necessary. The main indication for radiotherapy is in the treatment of oral tumours which, by virtue of their site or extent, are not amenable to surgical excision [17]. Cryosurgery may be indicated for lesions less than $2 \mathrm{~cm}$ in diameter that are fixed or minimally invasive into bone [18]. Larger lesions should generally be surgically resected. Oral cancer involving only soft tissues without bone involvement should be surgically excised and not frozen [18]. Finally, chemotherapy can be used as a palliative relief for disseminated oral tumors [12]. Chemotherapy protocols based on doxorubicin with or without vincristine and cyclophosphamide have been reported to have the best survival times for canine hemangiosarcoma [19]. Other promising potential therapies for canine hemangiosarcoma include the use of antibodies directed against specific antigens or receptors on tumor cell surfaces and the development of recombinant angiogenic inhibitors [19].

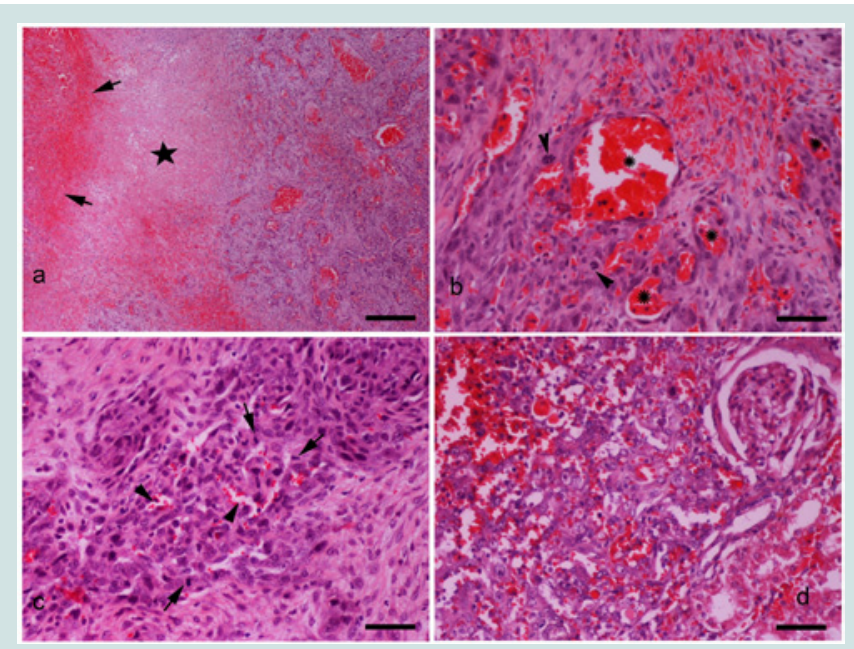

Figure 4: Primary mandibular mass. a. Numerous blood filled vascula channels of variable size (right half) neighboring to extensive hemorrhagic (arrows) and necrotic (star) areas.b. Abnormal vessels (asterisks) lined and surrounded by pleomorphic cells with spindloid to ovoid or polygonal shape, round to ovoid nucleus and usually two nucleoli. Note the mitotic figures (arrowheads). c. Area with small neoplastic vascular clefts and tumor cells with mitoses (arrows). A few tumor cells lie on a fairly dense fibrous stroma consisting of fibrosblasts and collagen fibers (upper left and lower right). d: renal metastasis. The kidney shows infiltration by a tumor histologically simila

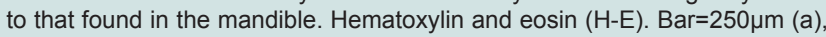
$50 \mu \mathrm{m}(\mathrm{b}, \mathrm{c}, \mathrm{d})$.

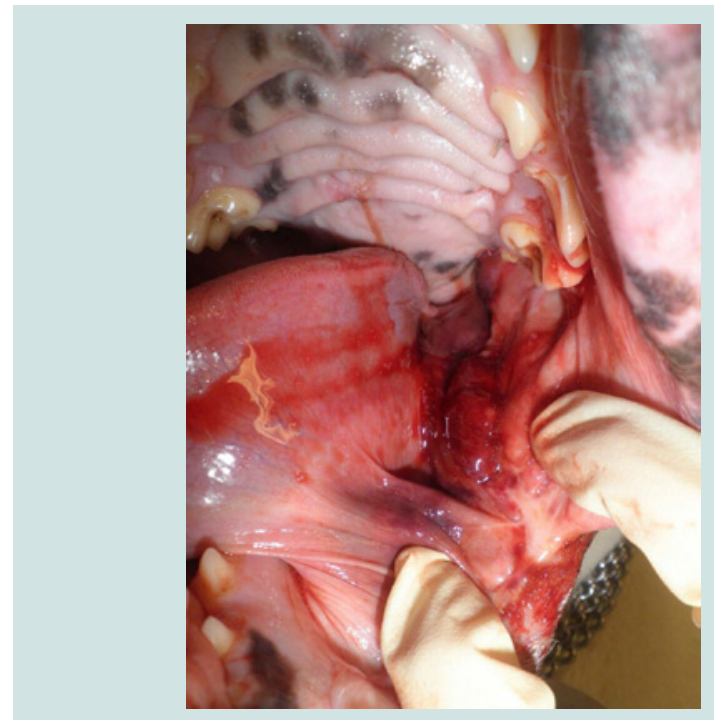

Figure 5: Recurrence of the neoplasm on the palatopharyngeal arch 5 months postoperatively.

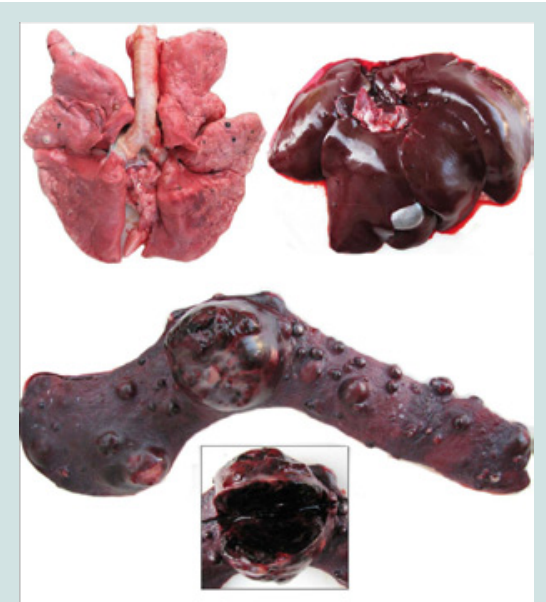

Figure 6: Grossly visible dark red spherical metastases in lungs, liver and spleen. The cut surface of a sizable multilobulate splenic mass shows multiple blood-filled spaces.

On presentation, the surgeons were not suspicious of hemangiosarcoma. The characteristics of the hemangiosarcoma presented here were: fast growth rate, relatively fast local recurrence and early development of multiorgan metastases. In this case, the histopathologic features of malignancy, like atypical mitoses and metastases combined with the evidence of blood within the majority of the neoplastic channels led us to the diagnosis of a rare mandibular hemangiosarcoma. Moreover, the absence of other tumor sites in the pre operative examination (radiography, ultrasonography), indicate that the oral cavity was the primary location.

The fact that pre-operative radiographs could not detect lung metastasis (multi-organ metastases) cannot exclude the possibility of milliary lung nodules presence, which are often not detectable radiographically. Moreover, the fact that only the mandibular lymph node was cytologically evaluated, probably underestimated the possibility of early metastasis. According to Herring and others [20], 
Citation: Papadimitriou SA, Anatolitou A, Brellou G, Kouki MI, Vlemmas I. Gingival Hemangiosarcoma of the Left Mandible in a Dog. J Veter Sci Med. 2014;2(1): 4

ISSN: $2325-4645$

excisional biopsy with histological evaluation of parotid, mandibular and medial retropharyngeal lymphnocentrums appears to provide greater information than traditional FNA assessment of mandibular lymph node only. Incisional biopsies and computed or magnetic resonance scans should be taken prior to surgery in order to develop the best treatment plan.

\section{References}

1. Thamm DH (2007) Miscellaneous tumors: Small Animal Clinical Oncology (4th Edition), Saunders Elsevier, St. Louis.

2. Hosgood G (1991) Canine hemangiosarcoma. Comp Contin Edu13:10651075.

3. Hammer AS, Couto CG (1992) Diagnosing and treating canine hemangiosarcoma. Vet Med 87: 188-201.

4. Ward H, Fox LE, Calderwood-Mays MB, Hammer AS, Couto CG (1994) Cutaneous hemangiosarcoma in 25 dogs: a retrospective study. J Vet Int Med 8: 345-348.

5. Brown NO, Patnaik AK, MacEwen EG (1985) Canine hemangiosarcoma: retrospective analysis of 104 cases. J Am Vet Med Assoc 186: 56-58.

6. Hargis AM, Ihrke PJ, Spangler WL, Stannard AA (1992) A retrospective clinicopathologic study of 212 dogs with cutaneous hemangiomas and hemangiosarcomas. Vet Pathol 29: 316-328.

7. Woods JP, Cuddon PA, Schenkman DI (1992) Prefrontal cortical and diencephalic cavernous hemangiosarcoma in a dog. Progress in Vet Neurology 3: 126-130.

8. Bingel SA, Brodey R, Sallen HL, Riser WH (1974) Hemangiosarcoma of bone in the dog. J Small Anim Pract 15: 303-322.
9. Pearson GR, Head KW (1976) Malignant hemangioendothelioma (angiosarcoma) in the dog. J Small Anim Pract 17: 737-745.

10. Oksanen A (1978) Hemangiosarcomas in dogs. J Comp Pathol 88: 585-595.

11. Arp LH, Grier PL (1984) Disseminated cutaneous hemangiosarcoma in a young dog. J Am Vet Med Assoc 185: 671-673.

12. Klausner JS, Hardy RM (1993) Alimentary tract, liver and pancreas. In: Textbook of Small Animal Surgery. (2nd Edition) W.B. Saunders Philadelphia, Pennsylvania.

13. Head KW, Else RW, Dubielzing RR (2002) Tumors and tumor like lesions of vascular tissue: Tumors of Domestic Animals. (4th Edition) lowa State Press.

14. Culbertson MR Jr (1982) Hemangiosarcoma of the Canine Skin and Tongue. Vet Pathol 19: 556-558.

15. Beck ER, Withrow SJ, McChesney AE, et al (1986) Canine tongue tumors: A retrospective review of 57 cases. J Am Anim Hosp Assoc 22: 525-532.

16. Remeeus P, Verbeek M (2002) Gingival hemangiosacroma in a dog. J Vet Dent 19: 25-26.

17. Morris J, Dobson J (2001) Head and neck: Small Animal Oncology. (1st Edition) Blackwell Science.

18. Withrow SJ, Vail DM, Page R (2007) Cancer of the gastrointestinal tract: Small Animal Clinical Oncology. (4th Edition) Saunders Elsevier, Missouri.

19. Clifford CA, Mackin AJ, Henry CJ, Beyond J (2000) Treatment of Canine Hemangiosarcoma. Vet Intern Med 14: 479-485.

20. Herring SE, Smith MM, Robertson JL (2002) Lymph node staging of oral and maxillofacial neoplasms in 31 dogs and cats. J vet Dent 19: 122-126. 